\title{
Research Progress on the Antitumor Effect of Polysaccharides from Fungus Used in Traditional Chinese Medicine
}

\author{
Ru Xiang, Bing Xu* \\ College of Medical Technology, Shaanxi University of Chinese Medicine, Xianyang 712046, Shaanxi Province, China \\ *Corresponding author: Bing Xu, xubing-95@163.com
}

\begin{abstract}
Cancer is a serious threat to human health. Fungal polysaccharide is a polar biological macromolecule with low toxicity. It has a wide range of biological activities, including immune regulation, antitumor activity, and antiviral activity. In recent years, the research results have shown that polysaccharides from fungus in traditional Chinese medicine have excellent antitumor effect in liver cancer, gastric cancer, breast cancer, colon cancer, esophageal cancer, and lung cancer. Therefore, the research and development of fungal polysaccharides are of great significance for the development of antitumor drugs in the future.
\end{abstract}

Keywords: Fungal polysaccharide; Traditional Chinese medicine; Antitumor; Action mechanism

Publication date: September 2021; Online publication: September 30, 2021

\section{Introduction}

Cancer, being a serious threat to human health, is the leading cause of death worldwide. Statistics have shown that 18.1 million new cases of cancer are expected in $2018^{[1]}$. Therefore, it is necessary to strengthen the research on tumor prevention and treatment to reduce the burden of cancer ${ }^{[2]}$.

Natural products are effective ways to develop new drugs. The statistics on the sources of clinical drugs show that more than $50 \%$ of clinical drugs are derived from natural products and their derivatives. Fungal polysaccharides have been widely used in the development of functional foods and health products ${ }^{[3]}$.

In recent years, research have suggested that the polysaccharides from fungus, including Grifola frondosa, Lentinus edodes, Auricularia auricula, and Inonotus obliquus, have good curative effect for various tumors. This research reviews the therapeutic effect of fungal polysaccharides through the research progress in recent years in regard to a few types of tumors.

\section{Liver cancer}

Dongxia LV and other researchers ${ }^{[4]}$ used Grifola frondosa polysaccharide with cisplatin in liver cancer, resulting in a significantly enhanced antitumor effect. A study ${ }^{[5]}$ have found that $58.3 \%$ of patients with liver cancer had significantly reduced symptoms after using Grifola frondosa polysaccharide. In another research ${ }^{[6]}$, it was found that the inhibitory rate of Ganoderma lucidum polysaccharide on tumor in hepatoma-bearing mice was dose-dependent. Other studies ${ }^{[7]}$ have shown that Cordyceps polysaccharide can significantly inhibit the growth of $\mathrm{H} 22$ tumors in mice. 


\section{Gastric cancer}

In a study that used chemotherapy and lentinan groups to treat patients with advanced gastric cancer ${ }^{[8]}$, the result showed that the number of cytotoxic $\mathrm{T}$ cells in the peripheral blood of patients increased significantly. Studies ${ }^{[9]}$ have found that using Poria cocos polysaccharide oral solution along with Western medicine can improve the quality of life and immune function of patients with advanced gastric cancer.

\section{Breast cancer}

It was found that letinous edodes polysaccharide, as an adjuvant chemotherapy in breast cancer, can effectively inhibit the growth of tumor blood vessels and regulate immune function ${ }^{[8]}$. It has also been found that letinous edodes polysaccharide can inhibit the expression of drug resistance genes (MDR1, MRP1, and BCRP), enhance the sensitivity of MCF-7 cells to taxol, as well as promote cell inhibition and apoptosis ${ }^{[10]}$. On the other hand, polysaccharides with the function of inhibiting MDA-MB-231 cell migration have been successfully isolated from Poria cocos ${ }^{[11]}$.

\section{Colon cancer}

It was found that the Inonotus obliquus polysaccharide can induce tumor cell apoptosis by affecting $\mathrm{Bax} / \mathrm{Bcl}-2$ ratio and activating caspase- ${ }^{[12]}$. In a research, it has been proven that Ganoderma lucidum polysaccharide can induce the apoptosis of colon cancer cells ${ }^{[12]}$. The apoptosis of LoVo cells is related to cell cycle arrest, a specific death receptor (Fas), mitochondrial pathway, and MAPK pathway. In addition, the apoptosis of HCT-116 cells is also related to cell cycle arrest, a specific death receptor (Fas) pathway, and mitochondrial pathway.

\section{Esophageal cancer}

Xiaolei Huo and other researchers ${ }^{[13]}$ found that Lentinus edodes polysaccharide plus taxol can significantly increase the apoptosis of esophageal cancer cells as well as inhibit the cell adhesion and migration related to tumor metastases.

\section{Lung cancer}

Grifola frondosa $\beta$ Polysaccharides can induce the apoptosis of lung cancer cells by activating oxidative stress ${ }^{[14]}$. Grifola frondosa polysaccharide can inhibit the proliferation of A549 cells, where its mechanism is related to the dissipation of mitochondrial membrane potential ${ }^{[15]}$. Junqiang Qiu and other researchers ${ }^{[16]}$ found that the complex formed by polysaccharides from Auricularia auricula and cisplatin has excellent antitumor cell proliferation property.

\section{Conclusion and prospect}

Cancer is a major disease which threatens human health. Patients with cancer require long-term radiotherapy, chemotherapy, or surgery, which would harm to their bodies. Fungal polysaccharides have excellent antitumor effect, low toxicity, and minimal side effects, thus having inherent advantages in the research and development of antitumor drugs. Hence, it is worthwhile to further explore on polysaccharides from fungus used in traditional Chinese medicine for new drugs in the treatment of cancer.

This article reviewed the progress of fungal polysaccharides in the treatment of several tumors. Polysaccharides from Grifola frondosa, Lentinus edodes, Auricularia auricula, Inonotus obliquus, and other fungi can alleviate malignant tumors, such as liver cancer, stomach cancer, breast cancer, colon cancer, esophagus cancer, as well as lung cancer, through various mechanisms and in combination with other drugs. 
In conclusion, fungal polysaccharides, which play a unique role in inhibiting malignant tumors, are worth further discussion.

\section{Disclosure statement}

The authors declare that there is no conflict of interest.

\section{References}

[1] Lu Z, 2020, Research on Tumour AMPK Activation Promotes NK Cell Anti Tumour Immunity and Synergistically Enhances PD-L1 Blocking Therapy. University of Chinese Academy of Sciences (Shenzhen Institutes of Advanced Technology, Chinese Academy of Sciences).

[2] Chen XQ, Xing NN, Huang LH, et al., Study on the Antitumor Activity and Diversity of Halophilic Fungi. Guangxi Science, 27(05): 526-531.

[3] Dai R, Liu RM, Xiao JH, 2015, Research Progress on the Mechanism of Reducing Blood Glucose Effect of Polysaccharide from Fungus Traditional Chinese Medicine. China Journal of Chinese Materia Medica, 40(02): 174-179.

[4] Lv DX, Du W, Fan XY et al., 2014, Research on Antitumor Effect of Polysaccharide from Grifola Frondosa Combined with Cisplatin on H22 Hepatocellular Carcinoma Transplanted Mice. Chinese Journal of Gerontology, 34(8): 2165-2166.

[5] Gan CF, 2014, Advances in Studies on Grifola Frondosa and Its Pharmacological Effects. Edible and Medicinal Mushrooms, 22(5): 264-267, 281.

[6] Shen FH, Wen YG, Tang QJ, et al., 2020, Research Progress on Antitumor Activity and Mechanism of Ganoderma Lucidum. Strait Pharmaceutical Journal, 32(04): 8-12.

[7] Tian Y, Li WJ, Qian ZM, et al., 2017, Research Progress on Pharmacological Experiment and Clinical Study on Antitumor Activity of Ganoderma Lucidum. Journal of Shenyang Pharmaceutical University, 34(10): 943-950.

[8] Zhang J, Su C, Jia YJ, et al., 2019, Progress in Clinical Application of Lentinus Edodes Polysaccharide in Antitumor Therapy. Tianjin Journal of Traditional Chinese Medicine, 36(11): 1137-1140.

[9] Yang HJ, Tian D, Liu ZX, et al., 2017, Research on Effect of Poria Cocos Polysaccharide Oral Liquid Combined with Oxaliplatin + Capecitabine on Quality of Life and Immune Function in Patients with Advanced Gastric Cancer. Chinese Medical Innovations, 14(17): 23-26.

[10] Zhong J, Wang Z, Wu HB, 2020, Sensitization Effect of Lentinus Edodes Polysaccharide on Taxol Chemotherapy in Human Breast Cancer Cell Line MCF-7. Chinese Journal of Surgical Oncology, 12(03): 236-240.

[11] Hu K, Luo Q, Zhu XF, et al., 2019, Effect of Poria Cocos Polysaccharides on Migration of Human Breast Cancer MDA-MB-231 Cells and Its Mechanism. Chinese Journal of Gerontology, 39(21): 5316-5319.

[12] Wang YM, Xie MZ, Zhang CW, et al., 2019, Research Progress on the Related Application of Medicinal Edible Fungus Grifola Frondosa to Antitumor. Agricultural Products Processing, (03): 8386.

[13] Huo XL, Han LN, Pei Z, et al., 2017, Effects of Lentinus Edodes Polysaccharide Combined with Taxol on Biological Behavior of Esophageal Cancer Cells. Journal of Southeast University (Medical Science Edition), 36(06): 897-900. 
[14] Tao J, Niu JJ, Hao SL, et al., 2019, Grifola frondosa $\beta$ Polysaccharides Induce Apoptosis of Lung Cancer Cells by Activating Oxidative Stress. Journal of North Sichuan Medical College, 34(02): 182186.

[15] Ni YB, Zhang LX, Cui DP, et al., 2017, Effect of Grifola Frondosa Polysaccharide on Mitochondria of Human Lung Adenocarcinoma Cell Line A549. Chinese Journal of Gerontology, 37(03): 542-544.

[16] Qiu JQ, Miao JN, Zhang H, et al., 2019, Anti-proliferation Activity of Cisplatin Complex of Auricularia Auricula Polysaccharide against HeLa and LoVo Cells. Chinese Journal of Modern Applied Pharmacy, 36(14): 1729-1732. 